Wright State University

CORE Scholar

Physics Faculty Publications

Physics

$9-1-2002$

\title{
Representation of a Quantum Ensemble as a Minimal Set of Pure States
}

Thomas E. Skinner

Wright State University - Main Campus, thomas.skinner@wright.edu

Steffen J. Glaser

Follow this and additional works at: https://corescholar.libraries.wright.edu/physics

Part of the Physics Commons

\section{Repository Citation}

Skinner, T. E., \& Glaser, S. J. (2002). Representation of a Quantum Ensemble as a Minimal Set of Pure States. Physical Review A, 66 (3), 32112.

https://corescholar.libraries.wright.edu/physics/185

This Article is brought to you for free and open access by the Physics at CORE Scholar. It has been accepted for inclusion in Physics Faculty Publications by an authorized administrator of CORE Scholar. For more information, please contact library-corescholar@wright.edu. 


\title{
Representation of a quantum ensemble as a minimal set of pure states
}

\author{
Thomas E. Skinner* \\ Physics Department, Wright State University, Dayton, Ohio 45435 \\ Steffen J. Glaser ${ }^{\dagger}$ \\ Institut für Organische Chemie und Biochemie II, Technische Universität München, Lichtenbergstrasse 4, 85747 Garching, Germany
}

(Received 17 May 2002; published 25 September 2002)

\begin{abstract}
The density matrix $\rho$ for an $n$-level system is decomposed into the minimum number of pure states necessary to calculate physical observables. The corresponding physical system is first represented by a set $\mathcal{B}$ of $n$ pure states $\left|\beta_{i}\right\rangle$, together with their statistical weights. The time evolution of the system is therefore calculated as $\mathcal{B}(t)=U \mathcal{B}\left(t_{0}\right)$, with the propagator $U$ operating on each member of the set, in contrast to the more laborious $\rho(t)=U \rho\left(t_{0}\right) U^{\dagger}$. At least one of the states can be eliminated from the set by reducing its weight to zero via a simple transformation of the density matrix. When there are degenerate weights, the transformation is applied using the weight with the largest degeneracy. Thus, even if none of the original statistical weights are equal to zero, so that $\operatorname{rank}(\rho)=n$, the system can be described by a set of $m$ states with $m<n$. The resulting minimal set provides a concommitant increase in calculational efficiency that depends on the fractional degeneracy. The independent evolution of the states comprising the set also provides an inherent parallelism that can be readily utilized in numerical computations. In addition to efficiency and conceptual simplification, this decomposition can provide physical insight, since the explicit contribution of each pure state to the dynamics of the system is readily apparent.
\end{abstract}

DOI: $10.1103 /$ PhysRevA.66.032112

PACS number(s): 03.65.Ca, 02.70.-c

\section{INTRODUCTION}

The density matrix formalism provides a straightforward procedure for calculating the dynamics of quantum mechanical systems. It is especially useful for describing macroscopic systems characterized by incomplete information-a situation which arises more often than not. For example, the state of each constituent in a large ensemble of identical particles can rarely, if ever, be determined exactly. The density matrix embodies all that is relevant to know of the system from a statistical point of view and distills systems which would be intractably complex at the microscopic level down to manageable proportions.

For systems which evolve according to unitary transformations, we obtain a further simplification by using the explicit set of pure states which can be found to comprise a given (finite dimensional) density matrix. Most of the material follows the treatment in standard references [1-3]. The final result, if somewhat obvious, is nonetheless useful and does not, to the best of our knowledge, appear to be routinely employed. It can significantly reduce the labor of calculation and is likely to be most applicable to numerical computations. But it also provides for conceptual simplification and enables physical insight concerning the dynamics of pure states to be applied to the dynamics of macroscopic ensembles. Use of a minimal set of pure states in calculations was, in fact, motivated by physical arguments and preceded the formal development presented here, which starts with the density matrix.

To establish the context of the present work, we first re-

\footnotetext{
*Electronic address: thomas.skinner@wright.edu

†Electronic address: glaser@ch.tum.de
}

view the salient features of the density operator. Our supplemental result then follows immediately from this overview. We summarize the entire procedure and close with an example illustrating some of its advantages.

\section{SYNOPSIS OF THE DENSITY MATRIX}

For a system prepared in a pure state $|\psi\rangle$, the mean value expected for measurement of a quantum mechanical observable represented by operator $Q$ is

$$
\langle Q\rangle=\langle\psi|Q| \psi\rangle .
$$

However, information on the state of the system may only be sufficient to allow its description as an incoherent superposition of pure states $\left|\psi_{i}\right\rangle$, with a statistical weight $p_{i}$ associated with each state. For this mixed state, the standard expectation value of Eq. (1) is simply modified to include the average over the ensemble of $N$ pure states, giving

$$
\langle Q\rangle=\sum_{i=1}^{N} p_{i}\left\langle\psi_{i}|Q| \psi_{i}\right\rangle .
$$

The number of constituents (molecules, for example) comprising a macroscopic system, and, hence, the number of pure states $N$, can be astronomically large. There is then insufficient information to determine even the $\left|\psi_{i}\right\rangle$ exactly, although the weights would be simply $p_{i}=1 / N$. Since $Q$ does not depend on the summation index, one can extract a density operator

$$
\rho=\sum_{i=1}^{N} p_{i}\left|\psi_{i}\right\rangle\left\langle\psi_{i}\right|
$$


to obtain the equivalent formulation

$$
\langle Q\rangle=\operatorname{Tr}(Q \rho) .
$$

The operator Tr returns the trace (sum of diagonal elements) of its argument.

The density matrix is thus an average of the operators $\left|\psi_{i}\right\rangle\left\langle\psi_{i}\right|$, reflecting the (classical) statistical uncertainty in one's knowledge of the exact states $\left|\psi_{i}\right\rangle$. Expanding each $\left|\psi_{i}\right\rangle$ in an orthonormal basis reduces $\rho$ to its usual matrix representation in a basis for an $n$-level system. In practical applications, $n \ll N$ and the $n^{2}$ elements of $\rho$ are easily determined from measurable macroscopic (average) properties of the system, such as energy or polarization. Since the pure states evolve from time $t_{0}$ to time $t$, through the propagator $U$ for the system, according to

$$
\left|\psi_{i}(t)\right\rangle=U\left(t, t_{0}\right)\left|\psi_{i}\left(t_{0}\right)\right\rangle,
$$

the time evolution of $\rho$ in Eq. (3) is given by

$$
\rho(t)=U\left(t, t_{0}\right) \rho\left(t_{0}\right) U^{\dagger}\left(t, t_{0}\right) .
$$

\section{PURE STATES COMPRISING THE DENSITY MATRIX}

The density operator is Hermitian and can always be represented as a diagonal matrix in the basis of its orthonormal eigenvectors $\left|\beta_{i}\left(t_{0}\right)\right\rangle$, if $\rho\left(t_{0}\right)$ is not diagonal to start with. The eigenvalues $\beta_{i}$, which are the probabilities that the corresponding states $\left|\beta_{i}\left(t_{0}\right)\right\rangle$ are occupied, satisfy $0 \leqslant \beta_{i} \leqslant 1$ and $\Sigma_{i} \beta_{i}=1$. A prototype example for which the initial density matrix is diagonal is a system in thermal equilibrium. The states $\left|\beta_{i}\left(t_{0}\right)\right\rangle$ in that case are also eigenvectors of the Hamiltonian $\mathcal{H}$ and the $\beta_{i}$ are the relative populations of the energy levels. More generally, for any diagonalized density matrix, we have

$$
\rho\left(t_{0}\right)=\sum_{i=1}^{n} \beta_{i}\left|\beta_{i}\left(t_{0}\right)\right\rangle\left\langle\beta_{i}\left(t_{0}\right)\right|
$$

as in Eq. (3). However, now both the weights and the corresponding states are known exactly, and expectation values at any time can be calculated explicitly, using Eq. (2), as

$$
\langle Q(t)\rangle=\sum_{i=1}^{n} \beta_{i}\left\langle\beta_{i}(t)|Q| \beta_{i}(t)\right\rangle .
$$

This point has also been touched upon in the context of stochastic processes and the evolution of a single pure state $[4,5]$. Thus, Eq. (5) rather than Eq. (6) can be used to calculate the evolution of the system, reducing the number of numerical operations by as much as a factor of 2 when the set of pure states and their associated weights

$$
\mathcal{B}(t)=\left\{\beta_{i},\left|\beta_{i}(t)\right\rangle\right\}, \quad i=1, \ldots, n
$$

is used in place of $\rho(t)$. If the density matrix is not diagonal initially, the cost to diagonalize it can only be compensated in applications requiring repeated propagation over many time increments. Further significant increases in efficiency can be obtained in the area of dissipative, nonunitary processes [6], which is beyond the scope of the present article.

Subsequent calculation of expectation values for $n$ separate pure states using Eq. (8) gives back this factor of 2 compared to $\operatorname{Tr}[Q \rho(t)]$ in the most general case of arbitrary observable $Q$. However, in many applications, the desired physical observable is represented by a relatively sparse matrix. Analytical expressions can be derived for $\langle Q\rangle$ as a function of either general vector components of $\left|\beta_{i}\right\rangle$ or matrix elements of $\rho$. For example, if $Q$ is diagonal or is equal to a direct product of spin-(1/2) operators (only one nonzero element in each row and column), it is straightforward to show that the net numerical reduction for a two-level system is still significant and approaches the full factor of 2 quickly for $n>2$. Equation (4) eventually becomes more efficient than Eq. (8) if a sufficiently large number of expectation values for different operators is needed at a fixed time. In the previous example, an $n$-level system requires the calculation of expectation values for approximately $6 n$ different operators to surpass the efficiency of the pure-states method. Which method to use will depend on the particular application, but additional gains in efficiency are possible that favor the decomposition into pure states.

\section{MINIMUM SET OF PURE STATES}

The diagonalized density operator can always be written in the form

$$
\rho\left(t_{0}\right)=\rho^{\prime}\left(t_{0}\right)+\beta_{k} \mathbb{1}
$$

for any given weight $\beta_{k}$ times the identity operator 1 . Thus, both $\rho$ and $\rho^{\prime}$ evolve according to Eq. (6), so that Eq. (10) holds for all $t$, and either operator can be used to construct the set of weighted pure states. However, $\rho^{\prime}\left(t_{0}\right)$ consists of $n$ modified weights $\beta_{i}^{\prime}=\beta_{i}-\beta_{k}$, which reduces the weight of the state $\left|\beta_{k}\right\rangle$ to zero. Hence, it can be eliminated from the set $\mathcal{B}$. If there are degenerate weights, as arises in many practical situations, choosing the weight with the largest degeneracy $g_{\max }$ maximally reduces the set

$$
\mathcal{B}(t) \rightarrow \mathcal{B}^{\prime}(t)=\left\{\beta_{i}^{\prime},\left|\beta_{i}^{\prime}(t)\right\rangle\right\}, \quad i=1, \ldots, m
$$

where $m=n-g_{\max }$. If all the weights are unique, $g_{\max }=1$. The modified weights $\beta_{i}^{\prime}$ satisfy $-1 \leqslant \beta_{i}^{\prime} \leqslant 1$ and, in general, no longer represent probabilities. Thus, $\rho^{\prime}$ is not strictly a density operator, although $\rho(t)$ can be reconstructed from $\rho^{\prime}(t)=\sum_{i=1}^{m} \beta_{i}^{\prime}\left|\beta_{i}^{\prime}(t)\right\rangle\left\langle\beta_{i}^{\prime}(t)\right|$ by adding the necessary multiple of 1 to obtain $\operatorname{Tr}(\rho)=1$. More formally, the system can be described by a set of $m<n$ states, even if none of the occupation numbers, $\beta_{i}$, are equal to zero, so that $\operatorname{rank}(\rho)$ $=n$. This result can be compared to a procedure for generating equivalent realizations of a given density matrix [7], in which the number of states, $k$, in any of the corresponding ensembles satisfies $k \geqslant \operatorname{rank}(\rho)$.

This smaller set can be efficiently used in calculations. For example, if an operator $Q$ is traceless, then $\langle Q\rangle$ $=\operatorname{Tr}(Q \rho)=\operatorname{Tr}\left(Q \rho^{\prime}\right)$ by Eq. (10), so the set $\mathcal{B}^{\prime}$ gives the same result as the set $\mathcal{B}$ when used in Eq. (8). More gener- 
(a)

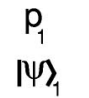

(b)

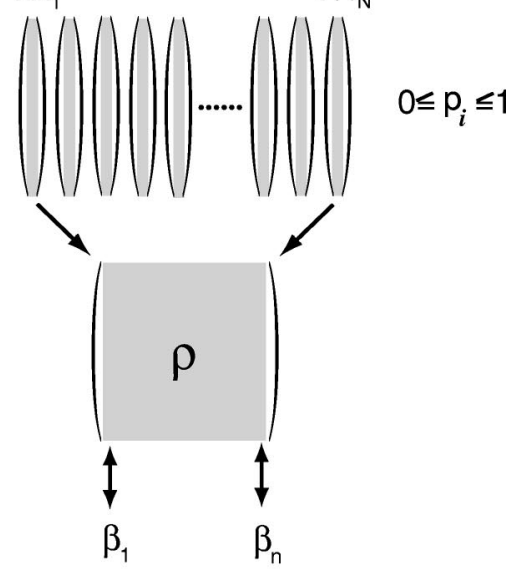

(c)

(d) $|\beta\rangle_{1}$

$|\beta\rangle_{n}$
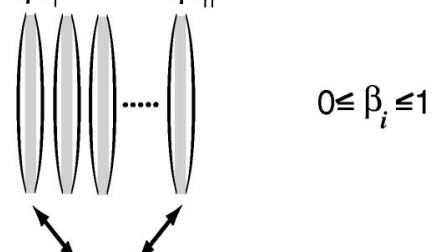

$-1 \leq \beta_{i}^{\prime} \leq 1$

$\beta_{1}^{\prime} \beta_{\mathrm{m}}^{\prime}$

$|\beta\rangle_{1}|\beta\rangle_{m}$

FIG. 1. (a) A macroscopic ensemble of $N$ noninteracting quantum systems is representable, in principle, as a mixture of $N$ pure states $\left|\psi_{i}\right\rangle$ together with the probabilities $p_{i}=1 / N$ for being in each state. (b) For a system with $n \ll N$ levels, an average over the ensemble according to Eq. (3) provides a statistical description of the system in terms of an $n \times n$ density matrix. (c) The density matrix is, in turn, representable by an easily determined set $\mathcal{B}$ of $n$-noninteracting pure states $\left|\beta_{i}\right\rangle$ with statistical weights $\beta_{i}$. (d) The set $\mathcal{B}$ can be reduced to the minimal set $\mathcal{B}^{\prime}$ consisting of $m$ $<n$ [Eq. (11)] pure states $\left|\beta_{i}^{\prime}\right\rangle$ with modified weights $\beta_{i}^{\prime}$ [Eq. (10)], including the case $n=\operatorname{rank}(\rho)$.

ally, if $\operatorname{Tr}[Q] \neq 0$, then $\operatorname{Tr}(Q \rho)-\operatorname{Tr}\left(Q \rho^{\prime}\right)=\beta_{k} \operatorname{Tr}(Q)$, and one merely adds this constant to the result obtained using the set $\mathcal{B}^{\prime}$. For an $n$-level system, the additional efficiency in calculating the time evolution of the set $\mathcal{B}^{\prime}(t)$ is $n /(n$ $\left.-g_{\max }\right)$, for an overall efficiency of a factor $2 n /\left(n-g_{\max }\right)$ compared to the product of the three matrices in Eq. (6).

\section{SUMMARY}

A schematic which summarizes the procedure is shown in Fig. 1. Fundamentally, quantum mechanics governs the dynamics of pure states (a). However, the exact state of each of the $N$ constituents comprising a macroscopic physical system cannot be realistically determined. The density matrix (b) represents a statistical description of what is known and pro-

vides a straightforward formalism for calculating system dynamics. But the identical density matrix can be constructed from a completely specified set of $n \ll N$ noninteracting pure states (c), and there can be advantages in returning conceptually to represent the system dynamics in terms of these states. Moreover, at least one state (and more, if there are degenerate weights) can be eliminated to form the minimal set (d) via Eq. (10).

Viewed this way, the density matrix is a device to ensure that the explicit set of pure states behaves incoherently, i.e., there is no interference among states in the calculation of expectation values using the trace relation in Eq. (4). Since Eq. (8) achieves this more directly, the density matrix represents unnecessary calculational overhead in many cases. In addition, each state in the weighted sets evolves independently, and its precise contribution to the observed quantity of interest is readily apparent. The parallelism inherent in this decomposition can be further exploited to increase the efficiency of numerical calculations.

\section{EXAMPLE}

To illustrate, we consider a macroscopic ensemble of quantum systems, in which each system is composed of two spin-(1/2) nuclei, $\mathbf{I}_{1}$ and $\mathbf{I}_{2}$, of the same isotopic species, in thermal equilibrium at temperature $T$ in an external magnetic field $B_{0}$. The initial density matrix is then diagonal, with eigenstates of the Hamiltonian populated according to the Boltzmann distribution. The field is considered sufficiently strong so that the Zeeman interaction is the dominant term in $\mathcal{H}=-\hbar \gamma B_{0}\left(I_{1 z}+I_{2 z}\right)$, with $\gamma$ equal to the gyromagnetic ratio of the spins. For all but cryogenic temperatures, fields available in the laboratory satisfy $\hbar \gamma B_{0} \ll k T$, and the partition function $Z(T)$ is to good approximation equal to the number of energy levels. Defining $p=\hbar \gamma B_{0} / k T$ gives

$$
\begin{aligned}
\rho & =e^{-\mathcal{H} / k T / Z(T)} \\
& \approx \frac{1}{4}(1-\mathcal{H} / k T) \\
& =\frac{1}{4} 1+\frac{p}{4}\left(I_{1 z}+I_{2 z}\right)=\frac{1}{4}\left(\begin{array}{cccc}
1+p & 0 & 0 & 0 \\
0 & 1 & 0 & 0 \\
0 & 0 & 1 & 0 \\
0 & 0 & 0 & 1-p
\end{array}\right) .
\end{aligned}
$$

Thus, the set $\mathcal{B}$ consists of four elementary unit vectors $\left|\beta_{i}\right\rangle$ (i.e., all components in each $\left|\beta_{i}\right\rangle$ equal to zero, except the $i$ th element $)$ with respective weights $\beta_{i}=(1$ $+p) / 4,1 / 4,1 / 4,(1-p) / 4$. Only three of the four weights are unique, and the states with $\beta_{2}=\beta_{3}$ can be eliminated via Eq. (10), to obtain the minimal set

$$
\begin{aligned}
\mathcal{B}^{\prime} & =\left\{\frac{p}{4},\left(\begin{array}{l}
1 \\
0 \\
0 \\
0
\end{array}\right) ; \quad-\frac{p}{4},\left(\begin{array}{l}
0 \\
0 \\
0 \\
1
\end{array}\right)\right\} \\
& =\left\{\frac{p}{4}, \quad|+z,+z\rangle ; \quad-\frac{p}{4}, \quad|-z,-z\rangle\right\}
\end{aligned}
$$


in terms of the usual $z$ basis for each spin. A rotation $U$ $=\exp \left[-i(\pi / 2) I_{1 y}\right]$ of $\mathbf{I}_{1}$ about the $y$ axis then gives for $\left\langle I_{1 x}\right\rangle$, according to Eq. (8),

$$
\begin{gathered}
\mathcal{B}^{\prime} \rightarrow U \mathcal{B}^{\prime}=\left\{\frac{p}{4},|+x,+z\rangle ;-\frac{p}{4},|-x,-z\rangle\right\}, \\
I_{1 x}| \pm x\rangle= \pm \frac{1}{2}| \pm x\rangle, \\
\left\langle I_{1 x}\right\rangle=\frac{p}{4}\left[\frac{1}{2}-\left(-\frac{1}{2}\right)\right]=\frac{p}{4} .
\end{gathered}
$$

The same result obtained using Eq. (4), ignoring the noncontributing term $1 / 4$, proceeds according to

$$
\begin{gathered}
\rho \rightarrow U \rho U^{\dagger}=\frac{p}{4}\left(I_{1 x}+I_{2 z}\right), \\
\operatorname{Tr}\left[I_{1 x} \rho(t)\right]=\frac{p}{4} \operatorname{Tr}\left[I_{1 x}^{2}\right]=\frac{p}{4} .
\end{gathered}
$$

There is therefore some redundancy in the representation of the original system by the density matrix of Eq. (12). This system is equivalent to a mixture of two noninteracting pure states with weights as given in Eq. (13). All the dynamics resides in these two states, with the other two supplying a static contribution. More generally (but limited to unitary transformations), the ensemble at the level of Fig. 1(c) always has at least one state, which can be arbitrarily assigned, that contributes nothing to the dynamical behavior of the system.

As noted after Eq. (11), the weights for the minimal set of pure states are no longer probabilities. The well-known dynamics of pure states subjected to a rotation allows the result of Eq. (14a) to be obtained by inspection, but the example serves to illustrate the factor of 4 difference in computational requirements of the two methods, in cases when the indicated matrix multiplications must be performed at each step.
In addition, for computer applications, the individual calculations involving each pure state in Eqs. (14) can be performed simultaneously on separate processors and the results combined in the final step of Eq. (14c).

\section{CONCLUSION}

We have considered the (unitary) time evolution of an ensemble of noninteracting quantum systems represented by an incoherent superposition of pure states. Although such mixed states are readily described by the density-matrix formalism, any $n \times n$ density matrix can be decomposed into an explicit set of $n$ pure states, together with their statistical weights. The new result presented here is that at least one of the states can always be eliminated from the set by reducing its weight to zero via the transformation in Eq. (10). When there are degenerate weights, the transformation is applied using the weight with the largest degeneracy, $g_{\max }$. The resulting minimal set consists of $m<n$ pure states, with $m$ $=n-g_{\max }$, even when $n=\operatorname{rank}(\rho)$. If all the weights are unique, $g_{\max }=1$. This set can be utilized directly in calculations of system dynamics, reducing the required number of numerical operations. Since each state evolves independently of the others, parallel computation can provide further efficiencies. Finally, the decomposition of the density matrix presented here (i.e., into the minimal set of noninteracting pure states necessary to calculate physical observables) can be conceptually simpler, enabling physical insight concerning the dynamics of a reduced set of pure states to be applied to the dynamics of macroscopic ensembles.

\section{ACKNOWLEDGMENTS}

The authors thank Christopher Fuchs for helpful discussion of the manuscript. T.E.S. was supported by The Wright State University while on sabbatical leave (2001-2002 academic year) at Technische Universität München. S.J.G. acknowledges support from the DFG (G1203/4-1) and the Fonds der Chemischen Industrie.
[1] R. C. Tolman, The Principles of Quantum Statistical Mechanics (Clarendon Press, Oxford, 1938).

[2] J. von Neumann, Mathematical Foundations of Quantum Mechanics (Princeton University Press, Princeton, 1955).

[3] U. Fano, Rev. Mod. Phys. 29, 74 (1957).

[4] J. Dalibard, Y. Castin, and K. Mølmer, Phys. Rev. Lett. 68, 580 (1992).
[5] R. Dum, P. Zoller, and H. Ritach, Phys. Rev. A 45, 4879 (1992).

[6] H. Carmichael, An Open Systems Approach to Quantum Optics (Springer-Verlag, Berlin, 1993).

[7] L. P. Hughston, R. Jozsa, and W. K. Wooters, Phys. Lett. A 183, 14 (1993). 